\title{
The negative impact of the COVID-19 lockdown on pain and physical function in patients with end-stage hip or knee osteoarthritis
}

\author{
Franz Endstrasser $^{1} \cdot$ Matthias Braito $^{1}$ (I) $\cdot$ Markus Linser $^{1} \cdot$ Anna Spicher $^{2} \cdot$ Moritz Wagner $^{1} \cdot$ Alexander Brunner $^{1}$
}

Received: 22 May 2020 / Accepted: 11 June 2020 / Published online: 18 June 2020

(c) European Society of Sports Traumatology, Knee Surgery, Arthroscopy (ESSKA) 2020

\begin{abstract}
Purpose The purpose of this study was to evaluate pain, functional impairment, mental health, and daily activity in patients with end-stage hip and knee osteoarthritis (OA) during the COVID-19 lockdown.

Methods The study included 63 patients, with hip or knee OA, who had been scheduled for arthroplasty that was postponed because of COVID-19. Patients were evaluated by telephone interviews during the first week after lockdown, in the fourth week, and again at the end of the lockdown. Patients rated their pain level on the basis of a visual analog scale (VAS) and completed WOMAC, SF-12 and Tegner activity scale (TAS) questionnaires.

Results VAS and WOMAC scores increased significantly during lockdown, while physical activity significantly decreased. At the final evaluation, VAS and WOMAC showed a significant negative correlation with TAS. The SF-12 subscale scores showed a significant decrease of the physical component during the lockdown, while the mental component remained largely unchanged. Patients with knee OA showed a faster progress of pain compared to those with hip OA. 50 patients (79\%) stated they wished to have arthroplasty as soon as possible.

Conclusion The COVID-19 lockdown had a significant impact on pain, joint function, physical function, and physical activity in patients with end-stage hip and knee OA.
\end{abstract}

Level of evidence II (Prospective cohort study).

Keywords COVID-19 $\cdot$ Arthroplasty $\cdot$ Osteoarthritis, hip $\cdot$ Osteoarthritis, knee $\cdot$ Physical functional performance $\cdot$ Mental health

\begin{tabular}{ll}
\multicolumn{2}{l}{ Abbreviations } \\
COVID-19 & Coronavirus disease 2019 \\
OA & Osteoarthritis \\
SD & Standard deviation \\
SF-12 & Short form 12 \\
TAS & Tegner activity scale \\
THA & Total hip arthroplasty \\
TKA & Total knee arthroplasty
\end{tabular}

Electronic supplementary material The online version of this article (https://doi.org/10.1007/s00167-020-06104-3) contains supplementary material, which is available to authorized users.

Matthias Braito

matthias.braito@gmail.com

1 Department of Orthopaedic and Trauma Surgery, BKH St. Johann in Tirol, Bahnhofstrasse 14, 6380 St. Johann in Tirol, Austria

2 Department of Trauma Surgery, Medical University of Innsbruck, Anichstrasse 35, 6020 Innsbruck, Austria

\author{
VAS Visual analog scale \\ WOMAC Western Ontario and McMaster Universities \\ Arthritis Index
}

\section{Introduction}

On March 11th, 2020, the World Health Organization (WHO) declared COVID-19 a pandemic. To minimize the rate of new infections and to prevent health institutions from capacity overload, most European countries started a complete national lockdown including closure of all nonessential businesses. To preserve medical resources, most elective orthopaedic surgical procedures across Europe were postponed, including total hip (THA) and total knee arthroplasty (TKA) [7, 13, 21].

Now that restrictions are started to be lifted, orthopaedic surgeons across Europe are preparing to restart arthroplasty surgery [15]. However, it remains unclear how the COVID19 lockdown will affect daily clinical practice in the future 
[8]. In this context, new hygiene standards could result in a reduction of hospitals' surgical capacities, leading to even longer waiting times in the future [10].

To establish guidelines to adequately reorganize elective surgery in outpatient clinics and hospitals after the end of lockdown, not only economic and safety aspects but also patient-related factors including the development of patients' complaints while waiting for arthroplasty have to be considered $[8,16,18]$. Since no study has yet investigated the development of clinical symptoms of patients with end-stage hip and knee osteoarthritis (OA) waiting for arthroplasty surgery, the purpose of this study was to investigate the course of (1) pain, (2) functional impairment, (3) mental health, and (4) daily activities during the course of COVID-19 lockdown. The present study hypothesized that the COVID-19 lockdown will limit physical activity, which will consequently increase clinical symptoms and decrease physical and mental function in patients with end-stage hip and knee OA.

\section{Materials and methods}

This prospective study was approved by the Ethical Committee of the Medical University Innsbruck, Austria (Process no. 1098/2020).

Between March 16th and April 30th 2020, a total of 68 arthroplasty surgeries (42 total hip and 26 total knee replacements) were postponed in our department due to the COVID19 lockdown. During the first week of lockdown (March 16th-March 22nd 2020), orthopaedic surgery residents contacted the patients via phone and informed them about their postponement. Furthermore, patients were informed about the purpose of this study and were asked for their consent to participate. Patients who agreed to participate received the written consent form by mail, which they had to return within 5 days after the start of lockdown (March 20th 2020). Patients who could not be contacted by phone, who refused participation or did not return their written consent were excluded. According to these criteria, a total of 63 patients

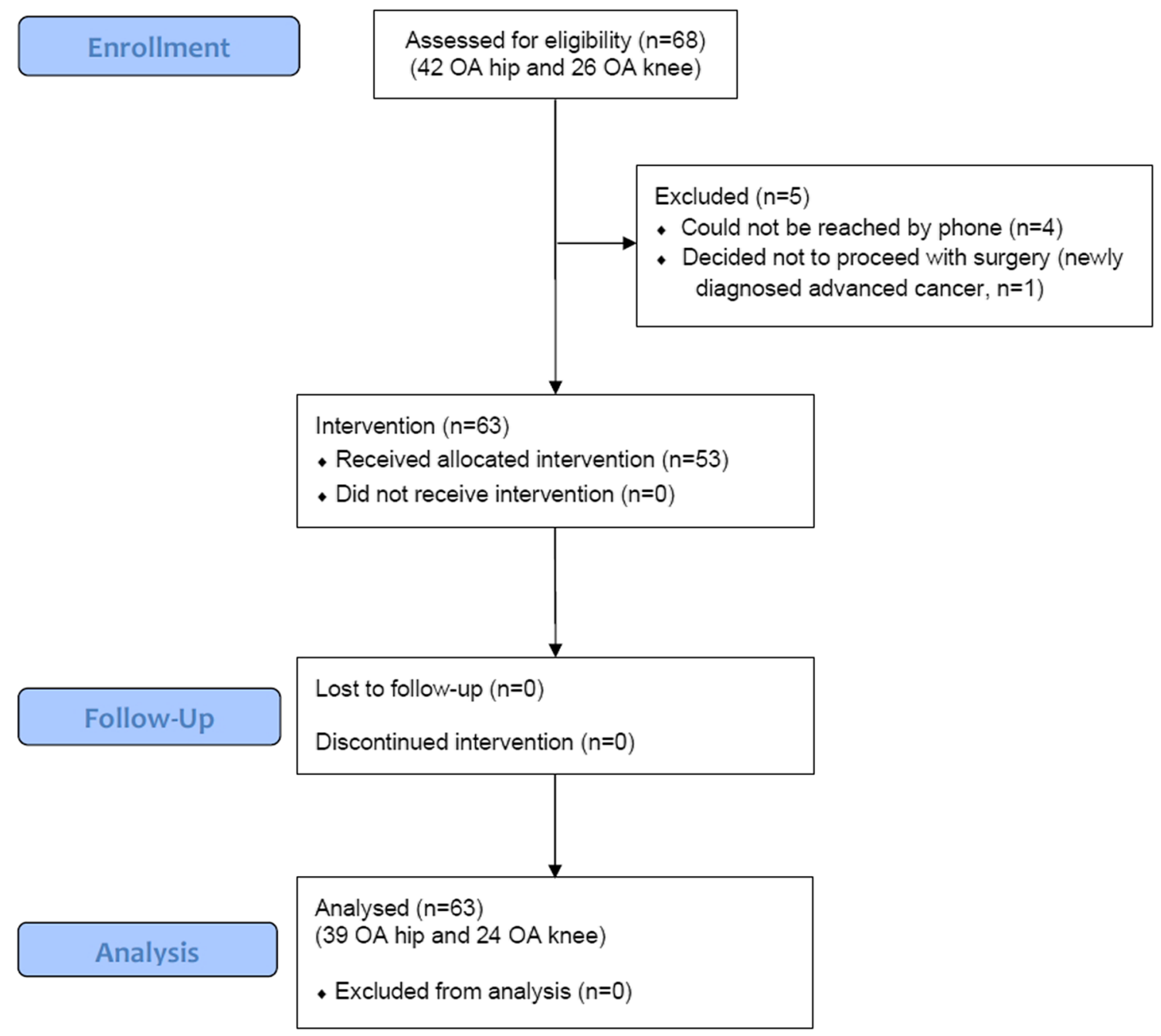

Fig. 1 Patient flow chart 
(28 men and 35 women) with 39 OA hips and 24 OA knees were available for further evaluation (Fig. 1). Of these, 28 (44\%) were females and 35 males (56\%). The mean age of participants was 62.4 years (range 26-86 years, standard deviation (SD): 11.84 years, Table 1). Patients waiting for THA were significantly younger (mean age: 59.7 years) than those waiting for TKA (mean age: 66.8 years). The mean BMI was $27.8 \mathrm{~kg} / \mathrm{m}^{2}$ (SD 4.5) with no significant difference between the groups. Indication for surgery was degenerative joint disease in all cases (four cases with hip dysplasia, one case with posttraumatic osteoarthritis of the hip). Most patients were assigned ASA (American Society of Anaesthesiologists) class 1 or 2 and more than half of our patients suffered from one or more comorbidities (Table 1). All patients included were asked to rate their hip- or knee-related pain on a visual analog scale (VAS) from 0 to 10 [11]. Furthermore, they completed a Western Ontario and McMaster Universities Osteoarthritis questionnaire (WOMAC) $[4,19]$ based on an 11-point numerical rating scale (from 0 to 10), the Short Form 12 (SF-12) [22, 23] and the Tegner activity scale (TAS) [20, 24].

The interviews were repeated in the fourth week of lockdown (April 6th-April 12th 2020) and after the end of lockdown (May 4th-May 8th 2020). The observers were blinded for the previous results. During the final interview, patients were also asked if they currently had reservations regarding in-patient treatment, and whether they preferred their

Table 1 Demographic and clinical characteristics of the study population

\begin{tabular}{|c|c|c|c|c|c|c|c|}
\hline & \multicolumn{2}{|c|}{ All patients $(N=63,100 \%)$} & \multicolumn{2}{|c|}{$\begin{array}{l}\text { Total hip arthroplasty }(N=39, \\
61.90 \%)\end{array}$} & \multicolumn{2}{|c|}{$\begin{array}{l}\text { Total knee arthroplasty }(N=24 \text {, } \\
38.10 \%)\end{array}$} & \multirow[t]{2}{*}{$p^{*}$} \\
\hline & Mean \pm SD & Min-max & Mean \pm SD & Min-max & Mean \pm SD & Min-max & \\
\hline Age (years) & $62.40 \pm 11.84$ & $26-86$ & $59.67 \pm 11.31$ & $26-82$ & $66.83 \pm 11.53$ & $46-86$ & 0.018 \\
\hline \multirow[t]{2}{*}{ BMI $\left(\mathrm{kg} / \mathrm{m}^{2}\right)$} & $27.76 \pm 4.52$ & $19.78-37.56$ & $27.08 \pm 4.33$ & $19.79-36.52$ & $28.87 \pm 4.69$ & $20.57-37.56$ & 0.128 \\
\hline & & $N$ & $\%$ & $N$ & $\%$ & $N$ & $\%$ \\
\hline \multicolumn{8}{|l|}{ Gender } \\
\hline Female & \multicolumn{2}{|r|}{28} & 44.44 & 17 & 43.59 & 11 & 45.83 \\
\hline Male & \multicolumn{2}{|r|}{35} & 55.56 & 22 & 56.41 & 13 & 54.17 \\
\hline \multicolumn{8}{|l|}{ Indication } \\
\hline Primary OA & \multicolumn{2}{|r|}{58} & 92.06 & 34 & 87.18 & 24 & 100.00 \\
\hline Hip dysplasia & \multicolumn{2}{|r|}{4} & 6.35 & 4 & 10.26 & 0 & 0.00 \\
\hline Posttraumatic OA & \multicolumn{2}{|r|}{1} & 1.59 & 1 & 2.56 & 0 & 0.00 \\
\hline \multicolumn{8}{|c|}{ Kellgren Lawrence grade } \\
\hline 2 & \multicolumn{2}{|r|}{9} & 14.28 & 3 & 7.69 & 6 & 25.00 \\
\hline 3 & \multicolumn{2}{|r|}{42} & 66.67 & 29 & 74.36 & 13 & 54.17 \\
\hline 4 & \multicolumn{2}{|r|}{12} & 19.05 & 7 & 17.95 & 5 & 20.83 \\
\hline ASA classification & & & & & & & \\
\hline 1 & & 16 & 25.40 & 11 & 28.21 & 5 & 20.83 \\
\hline 2 & & 35 & 55.56 & 22 & 56.41 & 13 & 54.17 \\
\hline 3 & & 4 & 6.35 & 1 & 1.59 & 3 & 12.50 \\
\hline Unknown & & 8 & 12.70 & 5 & 12.82 & 3 & 12.50 \\
\hline Co-morbidities & & & & & & & \\
\hline Hypertension & & 35 & 55.56 & 20 & 51.28 & 15 & 62.50 \\
\hline Cardiovascular & & 15 & 23.81 & 9 & 23.08 & 6 & 25.00 \\
\hline Cerebrovascular & & 5 & 7.94 & 1 & 2.56 & 4 & 16.67 \\
\hline COPD & & 5 & 7.94 & 3 & 7.69 & 2 & 8.33 \\
\hline Diabetes & & 8 & 12.70 & 3 & 7.69 & 5 & 20.83 \\
\hline Unknown & & 8 & 12.70 & 5 & 12.82 & 3 & 12.50 \\
\hline Prefers to do surger & $y$ as soon as po & sible & & & & & \\
\hline Yes & & 50 & 79.37 & 30 & 76.92 & 20 & 83.33 \\
\hline No & & 13 & 20.63 & 9 & 23.08 & 4 & 16.67 \\
\hline
\end{tabular}

$B M I$ body mass index, $A S A$ American Society of Anaesthesiologists, $N$ number, $S D$ standard deviation, Min minimum, Max maximum, $\mathrm{kg}$ kilogram, $m^{2}$ unit square meter, $O A$ osteoarthritis, $C O P D$ chronic obstructive pulmonary disease

${ }^{*} p$ value of independent $t$ test (age, BMI) for inter-group (THA/TKA) differences of distribution; $p$ value $<0.05$ significant (bold font) 
Table 2 Pre-, during- and post-COVID-19 lockdown clinical measurements from all patients waiting for arthroplasty surgeries

\begin{tabular}{|c|c|c|c|c|c|}
\hline & \multicolumn{3}{|c|}{ All patients $(N=63)$} & \multicolumn{2}{|c|}{ Comparison of distribution with } \\
\hline & Mean \pm SD & Median & Min-max & $\begin{array}{l}\text { Pre-lockdown } \\
\text { ( } p \text { value) }\end{array}$ & During ( $p$ value) \\
\hline VAS (pre) & $5.95 \pm 1.78$ & 6 & $2-10$ & & \\
\hline VAS (during) & $6.54 \pm 1.79$ & 7 & $2-10$ & $0.009 * *$ & \\
\hline VAS (post) & $6.59 \pm 2.21$ & 7 & $2-10$ & $<0.001 * *$ & $0.029 * *$ \\
\hline WOMAC (pre) & $43.37 \pm 12.38$ & 42.92 & $8.75-75.00$ & & \\
\hline Pain & $9.03 \pm 3.21$ & 8.33 & $0.83-16.25$ & & \\
\hline Stiffness & $3.73 \pm 1.88$ & 4.17 & $0.00-7.08$ & & \\
\hline Physical function & $30.61 \pm 9.04$ & 30.42 & $4.58-53.55$ & & \\
\hline WOMAC (during) & $47.11 \pm 14.62$ & 48.75 & $9.17-76.67$ & $0.004 *$ & \\
\hline Pain & $9.93 \pm 3.42$ & 10.00 & $0.83-17.92$ & $0.007 *$ & \\
\hline Stiffness & $3.88 \pm 2.19$ & 4.17 & $0.00-7.50$ & $0.324 *$ & \\
\hline Physical function & $33.31 \pm 10.86$ & 33.33 & $5.42-59.17$ & $0.005 *$ & \\
\hline WOMAC (post) & $53.99 \pm 16.39$ & 56.67 & $17.92-83.75$ & $<0.001 *$ & $<0.001 *$ \\
\hline Pain & $11.38 \pm 3.66$ & 12.08 & $2.92-18.33$ & $<0.001 *$ & $<0.001 *$ \\
\hline Stiffness & $5.61 \pm 1.77$ & 5.83 & $1.25-7.92$ & $<0.001 *$ & $<0.001 *$ \\
\hline Physical function & $38.37 \pm 12.15$ & 39.17 & $10.42-62.08$ & $<0.001 *$ & $<0.001 *$ \\
\hline SF-12 PCS (pre) & $37.89 \pm 8.92$ & 36.13 & $20.16-56.68$ & & \\
\hline SF-12 PCS (during) & $37.36 \pm 9.08$ & 36.13 & $20.16-56.68$ & $0.204 * *$ & \\
\hline SF-12 PCS (post) & $35.48 \pm 9.62$ & 34.54 & $18.83-52.88$ & $0.026 * *$ & $0.071 * *$ \\
\hline SF-12 MCS (pre) & $59.81 \pm 6.76$ & 61.66 & $38.97-69.32$ & & \\
\hline SF-12 MCS (during) & $59.24 \pm 6.77$ & 60.81 & $38.97-69.04$ & $0.130 * *$ & \\
\hline SF-12 MCS (post) & $59.49 \pm 5.45$ & 60.02 & $48.73-69.41$ & $0.929 * *$ & $0.755^{* *}$ \\
\hline TAS (pre) & n.r. & 3 & $0-5$ & & \\
\hline TAS (during) & n.r. & 2 & $0-5$ & $0.046 * *$ & \\
\hline TAS (post) & n.r. & 2 & $0-5$ & $0.017 * *$ & $0.059 * *$ \\
\hline
\end{tabular}

$N$ number, $S D$ standard deviation, Min minimum, Max maximum, VAS Visual Analog Scale, WOMAC Western Ontario And McMaster Universities Osteoarthritis Index, SF-12 short-form health survey, PCS physical component summary, MCS mental component summary, TAS Tegner Activity Scale, n.r. not reported

*Paired samples $t$ test

**Wilcoxon Signed Rank test; $p$ value $<0.05$ significant (bold font) surgery to be re-scheduled as soon as possible or if they preferred a further delay.

\section{Statistical methods}

Initially, a Shapiro-Wilk analysis was performed to test linear data for normal distribution. Mean WOMAC scores were compared using a paired sample $t$ test. Results from the VAS scales, the SF-12 and the TAS were compared with a Wilcoxon signed-rank test. A Mann-Whitney $U$ test was applied to test for equality of distribution of VAS, SF-12 and TAS. Furthermore, a Spearman Rank-Order analysis was performed to calculate the correlation between TAS, VAS and WOMAC. $p$ values of $<0.05$ were considered statistically significant.

\section{Results}

The mean VAS scores increased significantly between the first and second and between the second and final interviews (Table 2). Likewise, the mean WOMAC scores increased continuously during the lockdown. The SF-12 physical component summary scores (SF-12 PCS) decreased significantly between the first and the last interview (Table 2). In contrast, the SF-12 mental component summary scores (SF-12 MCS) showed no significant difference between the measurements. The Tegner activity scale (TAS) decreased significantly during lockdown (Table 2). The majority of our patients (79\%) preferred to have surgery as soon as possible, while the remaining $21 \%$ wished to further delay their treatment until after the end of the COVID-19 pandemic (Table 1). 
Table 3 The correlations between physical activity and clinical measurements

\begin{tabular}{|c|c|c|c|c|c|c|}
\hline \multicolumn{7}{|c|}{ Spearman's rank-order correlation with TAS } \\
\hline \multirow[t]{2}{*}{ Variable } & \multicolumn{2}{|c|}{ All patients } & \multicolumn{2}{|c|}{ Total hip arthroplasty } & \multicolumn{2}{|c|}{$\begin{array}{l}\text { Total knee arthro- } \\
\text { plasty }\end{array}$} \\
\hline & $r_{\mathrm{s}}$ & $p$ & $r_{\mathrm{s}}$ & $p$ & $r_{\mathrm{s}}$ & $p$ \\
\hline \multicolumn{7}{|l|}{ Before lockdown } \\
\hline VAS & -0.129 & 0.314 & -0.042 & 0.801 & -0.260 & 0.220 \\
\hline WOMAC & -0.135 & 0.291 & -0.032 & 0.849 & -0.152 & 0.479 \\
\hline Pain & 0.013 & 0.918 & 0.002 & 0.989 & 0.115 & 0.592 \\
\hline Stiffness & 0.022 & 0.862 & 0.121 & 0.464 & -0.026 & 0.906 \\
\hline Physical function & -0.180 & 0.158 & -0.066 & 0.691 & -0.270 & 0.201 \\
\hline SF-12 PCS & 0.353 & 0.005 & 0.381 & 0.017 & 0.300 & 0.154 \\
\hline SF-12 MCS & -0.066 & 0.608 & -0.205 & 0.211 & 0.191 & 0.371 \\
\hline \multicolumn{7}{|l|}{ During lockdown } \\
\hline VAS & -0.201 & 0.133 & -0.201 & 0.219 & -0.203 & 0.342 \\
\hline WOMAC & -0.268 & 0.034 & -0.248 & 0.128 & -0.259 & 0.222 \\
\hline Pain & -0.140 & 0.274 & -0.128 & 0.438 & -0.094 & 0.664 \\
\hline Stiffness & 0.003 & 0.983 & 0.014 & 0.933 & 0.075 & 0.728 \\
\hline Physical function & -0.286 & 0.023 & -0.233 & 0.154 & -0.329 & 0.116 \\
\hline SF-12 PCS & 0.431 & $<0.001$ & 0.565 & $<0.001$ & 0.245 & 0.249 \\
\hline SF-12 MCS & -0.017 & 0.895 & -0.124 & 0.453 & 0.204 & 0.338 \\
\hline \multicolumn{7}{|l|}{ Post lockdown } \\
\hline VAS & -0.470 & $<0.001$ & -0.506 & 0.001 & -0.486 & 0.016 \\
\hline WOMAC & -0.495 & $<0.001$ & -0.454 & 0.004 & $-\mathbf{0 . 5 2 0}$ & 0.009 \\
\hline Pain & -0.344 & 0.006 & -0.367 & 0.022 & -0.351 & 0.092 \\
\hline Stiffness & -0.433 & $<0.001$ & -0.473 & 0.002 & -0.421 & 0.040 \\
\hline Physical function & -0.481 & $<0.001$ & -0.466 & 0.003 & -0.489 & 0.015 \\
\hline SF-12 PCS & 0.663 & $<0.001$ & 0.677 & $<0.001$ & 0.546 & 0.006 \\
\hline SF-12 MCS & -0.030 & 0.816 & -0.313 & 0.052 & 0.489 & 0.015 \\
\hline
\end{tabular}

$r_{\mathrm{s}}$ Spearman's rank correlation coefficient; $p$ value $<0.05$ significant (bold font)

VAS Visual Analog Scale, WOMAC Western Ontario And McMaster Universities Osteoarthritis Index, SF12 short-form-health survey, PCS physical component summary, MCS mental component summary, TAS Tegner Activity Scale
Additional results attained by the correlations between physical activity, pain, physical and mental function (Table 3), the intra- and inter-group analysis for the hip and knee OA patients (Table 4), and the intra- and inter-group analysis of patients who prefer surgery as soon as possible compared to those who want to wait (Table 5) are given in the corresponding tables, respectively.

\section{Discussion}

The data from this study confirm our hypothesis that the COVID-19 lockdown significantly affects the level of physical activity, joint function and physical function in patients with advanced hip and knee OA. Mental health remained unaffected during the lockdown.

The difference in WOMAC scores between the beginning and the end of lockdown exceeds the minimum score difference that has been reported as clinically important [6]. This suggests a clinically relevant loss of joint function during the lockdown. Overall, patients with hip OA showed higher WOMAC scores compared to patients with knee OA. This phenomenon has been previously described in clinical trials with high volume samples [2, 4, 5]. VAS pain scores and TAS showed a significant deterioration during lockdown as well. However, observed changes of VAS and TAS were of limited clinical significance.

During lockdown, VAS and WOMAC scores showed an increasing correlation with the level of activity (TAS). 
Table 4 Pre-, during- and post-COVID-19 lockdown (intra- and inter-group analysis for hip and knee OA)

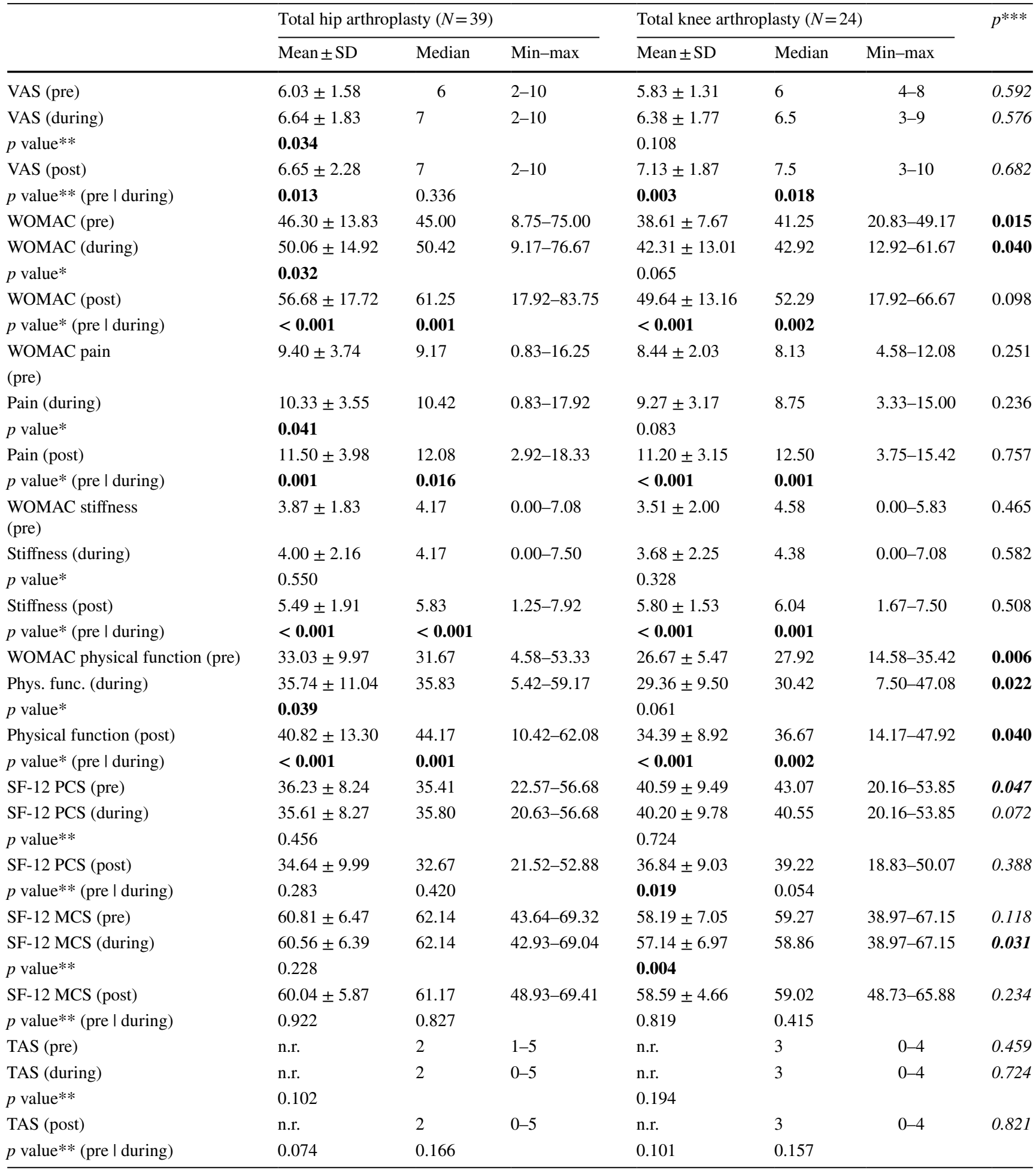

$S D$ standard deviation, Min minimum, Max maximum, VAS Visual Analog Scale, WOMAC Western Ontario And McMaster Universities Osteoarthritis Index, SF-12 short-form-health survey, PCS physical component summary, MCS mental component summary, TAS Tegner Activity Scale, n.r. not reported

*Comparison of the distribution of values pre-/during-/post-COVID-19 lockdown within the group using a paired $t$ test

**Comparison of the distribution of values pre-/during-/post-COVID-19 lockdown within the group using the Wilcoxon Signed-Rank test ***Comparison between the groups (prefers near time surgery: yes/no) using an independent $t$ test, respectively, the Mann-Whitney $U$ test for non-normal distributed results (italic font); $p$ value $<0.05$ significant (bold font) 
Table 5 Pre-, during- and post-COVID-19 lockdown inter-group analysis of patients who prefer near time surgical intervention compared to those who do not

\begin{tabular}{|c|c|c|c|c|c|c|c|}
\hline & \multicolumn{3}{|c|}{ Prefers near time surgery $(N=50)$} & \multicolumn{3}{|c|}{ Does not want near time surgery $(N=13)$} & \multirow[t]{2}{*}{$p^{* * *}$} \\
\hline & Mean \pm SD & Median & Min-max & Mean \pm SD & Median & Min-max & \\
\hline VAS (pre) & $6.08 \pm 1.44$ & 6 & $2-10$ & $5.46 \pm 1.56$ & 5 & $4-8$ & 0.133 \\
\hline VAS (during) & $6.80 \pm 1.73$ & 7 & $2-10$ & $5.54 \pm 1.76$ & 6 & $3-8$ & 0.026 \\
\hline$p$ value** & 0.002 & & & 0.943 & & & \\
\hline VAS (post) & $7.43 \pm 1.94$ & 8 & $2-10$ & $5.46 \pm 2.18$ & 6 & $2-8$ & 0.006 \\
\hline$p$ value $* *$ & $<0.001$ & 0.010 & & 0.904 & 0.829 & & \\
\hline WOMAC (pre) & $43.86 \pm 12.18$ & 43.33 & $8.75-72.50$ & $41.51 \pm 13.45$ & 38.75 & $28.75-75.00$ & 0.546 \\
\hline WOMAC (during) & $48.12 \pm 14.38$ & 49.17 & $9.17-76.67$ & $43.24 \pm 15.47$ & 40.00 & $20.00-75.00$ & 0.287 \\
\hline$p$ value* & 0.003 & & & 0.586 & & & \\
\hline WOMAC (post) & $56.12 \pm 14.53$ & 57.50 & $20.00-83.75$ & $45.83 \pm 20.85$ & 52.08 & $17.92-75.83$ & 0.043 \\
\hline$p$ value* (pre I during) & $<0.001$ & $<0.001$ (dur) & & 0.347 & 0.475 & & \\
\hline WOMAC pain (pre) & $9.18 \pm 3.18$ & 9.17 & $0.83-16.25$ & $8.46 \pm 3.40$ & 7.92 & $5.00-16.25$ & 0.475 \\
\hline Pain (during) & $10.18 \pm 3.40$ & 10.42 & $0.83-17.92$ & $8.94 \pm 3.48$ & 8.33 & $3.33-16.25$ & 0.248 \\
\hline$p$ value* & 0.008 & & & 0.505 & & & \\
\hline Pain (post) & $11.90 \pm 3.17$ & 12.29 & $4.58-18.33$ & $9.39 \pm 4.77$ & 10.42 & $2.92-16.67$ & $\mathbf{0 . 0 2 7}$ \\
\hline$p$ value* (pre I during) & $<0.001$ & $<0.001$ & & 0.371 & 0.552 & & \\
\hline WOMAC stiffness (pre) & $3.84 \pm 1.82$ & 4.38 & $0.00-7.08$ & $3.30 \pm 2.13$ & 2.92 & $0.00-6.67$ & 0.361 \\
\hline Stiffness (during) & $4.00 \pm 2.16$ & 4.38 & $0.00-7.50$ & $3.40 \pm 2.32$ & 2.92 & $0.00-6.67$ & 0.380 \\
\hline$p$ value* & 0.335 & & & 0.787 & & & \\
\hline Stiffness (post) & $5.83 \pm 1.59$ & 6.04 & $1.25-7.92$ & $4.78 \pm 2.22$ & 5.42 & $1.67-7.50$ & 0.056 \\
\hline$p$ value*(pre I during) & $<0.001$ & $<0.001$ & & 0.110 & 0.118 & & \\
\hline WOMAC physical function (pre) & $30.83 \pm 8.94$ & 30.83 & $4.58-53.33$ & $29.74 \pm 9.72$ & 29.17 & $15.83-52.08$ & 0.702 \\
\hline Physical function (during) & $33.93 \pm 10.76$ & 33.75 & $5.42-59.17$ & $30.90 \pm 11.35$ & 30.42 & $15.83-52.08$ & 0.374 \\
\hline$p$ value* & 0.004 & & & 0.615 & & & \\
\hline Physical function (post) & $39.82 \pm 10.95$ & 39.17 & $10.42-62.08$ & $32.82 \pm 15.21$ & 39.17 & $13.33-52.50$ & 0.064 \\
\hline$p$ value* (pre I during) & $<0.001$ & $<0.001$ & & 0.339 & 0.478 & & \\
\hline SF-12 PCS (pre) & $38.18 \pm 8.84$ & 36.63 & $20.16-56.68$ & $36.77 \pm 9.50$ & 36.03 & $22.57-53.85$ & 0.547 \\
\hline SF-12 PCS (dur.) & $37.61 \pm 9.04$ & 36.42 & $20.16-56.68$ & $36.40 \pm 9.55$ & 36.03 & $22.01-53.85$ & 0.671 \\
\hline$p$ value $* *$ & 0.186 & & & 0.893 & & & \\
\hline SF-12 PCS (post) & $34.40 \pm 9.29$ & 33.02 & $19.87-52.88$ & $39.59 \pm 10.14$ & 41.45 & $18.83-50.07$ & 0.077 \\
\hline$p$ value** (pre I during) & 0.004 & 0.012 & & 0.173 & 0.136 & & \\
\hline SF-12 MCS (pre) & $59.59 \pm 7.17$ & 60.81 & $38.97-69.32$ & $60.66 \pm 5.03$ & 61.84 & $51.70-67.15$ & 0.845 \\
\hline SF-12 MCS (dur.) & $58.70 \pm 6.98$ & 60.18 & $38.97-69.04$ & $61.33 \pm 5.63$ & 63.15 & $48.39-67.15$ & 0.197 \\
\hline$p$ value** & 0.006 & & & 0.889 & & & \\
\hline SF-12 MCS (post) & $59.70 \pm 5.68$ & 60.77 & $48.73-69.41$ & $58.68 \pm 4.56$ & 57.81 & $49.74-65.73$ & 0.552 \\
\hline$p$ value $* *$ (pre I during) & 0.761 & 0.332 & & 0.345 & 0.209 & & \\
\hline TAS (pre) & n.r. & 2 & $0-5$ & n.r. & 3 & $2-4$ & 0.101 \\
\hline TAS (during) & n.r. & 2 & $0-5$ & n.r. & 3 & $0-4$ & 0.261 \\
\hline$p$ value** & 0.132 & & & 0.180 & & & \\
\hline TAS (post) & n.r. & 2 & $0-5$ & n.r. & 3 & $0-4$ & 0.092 \\
\hline$p$ value $* *$ (pre I during) & 0.034 & 0.054 & & 0.257 & 1.000 & & \\
\hline
\end{tabular}

$S D$ standard deviation, Min minimum, Max maximum, VAS Visual Analog Scale, WOMAC Western Ontario And McMaster Universities Osteoarthritis Index, SF-12 short-form-health survey, PCS physical component summary, MCS mental component summary, TAS Tegner Activity Scale, n.r. not reported

*Comparison of the distribution of values pre-COVID-19 with during/post COVID-19 within the group using a paired $t$ test

**Comparison of the distribution of values pre-COVID-19 with during/post COVID-19 within the group using the Wilcoxon Signed Ranks test

***Comparison between the groups (total hip arthroplasty/total knee arthroplasty) using an independent $t$ test, respectively, the Mann-Whitney $U$ test for non-normal distributed results (italic font); $p$ value $<0.05$ significant (bold font) 
This supports our hypothesis that the continuous decrease of physical activity is associated with an increase in pain and loss of joint function. These findings are in accordance with the results of prior studies and demonstrate the importance of home exercise programs to decrease symptoms during a possible second lockdown [3, 12, 14]. Knee OA patients showed a faster deterioration in pain score compared to hip OA patients during lockdown, which suggests that loss of activity has a higher impact on OA knees.

A recent Chinese study showed that social isolation during the COVID-19 lockdown significantly affected the psychological health of the population in the Hubei province [1]. Consequently, the authors underlined the importance of mental health services and provisioning of psychiatric treatments during an elongated lockdown [1,9]. In contrast to these findings, the patients evaluated in our study did not show any deterioration of their mental status, which suggests that psychiatric assistance may be of minor importance in patients with hip and knee OA. However, our study was not designed to detect a significant difference of mental health and the SF-12 mental component summary score may not adequately assess mental criteria.

In the evaluated cohort, nearly $80 \%$ of patients stated that they desired surgery as soon as possible. Only $20 \%$ wished to further delay their treatment, mainly because they wished to wait until things could get back to normality again. Subgroup analysis showed that patients who preferred their surgery sooner suffered from significant pain and function decline during the lockdown; whereas, patients who preferred a further delay did not significantly deteriorate. This suggests that the patients' decision depended predominantly on the development of their symptoms and not on any reservations regarding in-house treatment.

Based on the findings of this study, the authors assume that lockdown not only affected patients with end-stage OA but also those with advanced OA, which could result in a higher request for arthroplasty surgery in Europe in the months to come [18].

However, our findings may not be generalizable: in countries without a national health service or governmental health insurance, economic crises are usually associated with increasing loss of commercial health insurance due to unemployment [16]. As result, many patients are unable to afford surgery. It must be expected that arthroplasty volume will decrease in these countries even though there is an increased demand [16, 17, 25].

The present study has several limitations. The relatively small sample size may inhibit adequate analysis of the physical function and mental condition of our cohort. Moreover, the absence of a control group limits the explanatory power of our results. There is a potential systemic bias since patients may have hoped for earlier appointments for surgery if they reported higher pain values. Furthermore, the authors are unable to determine if the observed deteriorations are reversible after the end of lockdown. However, further investigation of this question would raise ethical concerns as we are striving to treat patients suffering from severe pain as soon as possible.

\section{Conclusion}

The COVID-19 lockdown had a significant impact on pain, joint function, physical function, and physical activity in patients with end-stage hip and knee OA. Our results can be taken as a basis for the course of OA patients' complaints, if patient-related factors are considered when reorganizing arthroplasty surgery after the end of lockdown.

Acknowledgements The editors would like to thank Oxford science editing for providing professional language editing.

Author contributions E. F collected data, drafted the manuscript, performed statistical analysis, and acts as first author. B. M. drafted the manuscript and acts as corresponding author. L. M. collected data and acts as co-author. S. A. participated in the study design and acts as coauthor. W. M. collected data and acts as co-author. B. A. conceived of the study, generated its design, and acts as senior author.

\section{Compliance with ethical standards}

Conflict of interest The authors declare that they have neither financial nor non-financial competing interests.

Funding This research did not receive any specific grant from funding agencies in the public, commercial, or not-for-profit sectors. There is no funding source for this study.

Ethical approval This study was approved by the local ethics committee of the Medical University of Innsbruck. The approval reference number is 1098/2020. Research was performed in accordance with the Declaration of Helsinki.

Informed consent Informed consent was obtained from each subject prior to inclusion.

\section{References}

1. Ahmed MZ, Ahmed O, Aibao Z, Hanbin S, Siyu L, Ahmad A (2020) Epidemic of COVID-19 in China and associated psychological problems. Asian J Psychiatr 51:102092

2. Angst F, Ewert T, Lehmann S, Aeschlimann A, Stucki G (2005) The factor subdimensions of the Western Ontario and McMaster Universities Osteoarthritis Index (WOMAC) help to specify hip and knee osteoarthritis. a prospective evaluation and validation study. J Rheumatol 32:1324-1330

3. Anwer S, Alghadir A, Brismee JM (2016) Effect of Home Exercise Program in Patients With Knee Osteoarthritis: a Systematic Review and Meta-analysis. J Geriatr Phys Ther 39:38-48

4. Bellamy N, Buchanan WW, Goldsmith CH, Campbell J, Stitt LW (1988) Validation study of WOMAC: a health status 
instrument for measuring clinically important patient relevant outcomes to antirheumatic drug therapy in patients with osteoarthritis of the hip or knee. J Rheumatol 15:1833-1840

5. Canfield M, Savoy L, Cote MP, Halawi MJ (2020) Patientreported outcome measures in total joint arthroplasty: defining the optimal collection window. Arthroplast Today 6:62-67

6. Clement ND, Bardgett M, Weir D, Holland J, Gerrand C, Deehan DJ (2018) What is the minimum clinically important difference for the WOMAC index after TKA? Clin Orthop Relat Res 476:2005-2014

7. D'Apolito R, Faraldi M, Ottaiano I, Zagra L (2020) Disruption of arthroplasty practice in an orthopedic center in Northern Italy during the coronavirus disease 2019 pandemic. J Arthroplasty. https://doi.org/10.1016/j.arth.2020.04.057

8. de Caro F, Hirschmann TM, Verdonk P (2020) Returning to orthopaedic business as usual after COVID-19: strategies and options. Knee Surg Sports Traumatol Arthrosc 28:1699-1704

9. Duan L, Zhu G (2020) Psychological interventions for people affected by the COVID-19 epidemic. Lancet Psychiatry 7:300-302

10. Hirschmann MT, Hart A, Henckel J, Sadoghi P, Seil R, Mouton C (2020) COVID-19 coronavirus: recommended personal protective equipment for the orthopaedic and trauma surgeon. Knee Surg Sports Traumatol Arthrosc 28:1690-1698

11. Katz J, Melzack R (1999) Measurement of pain. Surg Clin North Am 79:231-252

12. Kraus VB, Sprow K, Powell KE, Buchner D, Bloodgood B, Piercy $\mathrm{K}$ et al (2019) Effects of physical activity in knee and hip osteoarthritis: a systematic umbrella review. Med Sci Sports Exerc 51:1324-1339

13. Liebensteiner MC, Khosravi I, Hirschmann MT, Heuberer PR, Board of the AGASoA, Joint S et al (2020) Massive cutback in orthopaedic healthcare services due to the COVID-19 pandemic. Knee Surg Sports Traumatol Arthrosc 28:1705-1711

14. Morcos MW, Teeter MG, Somerville LE, Lanting B (2020) Correlation between hip osteoarthritis and the level of physical activity as measured by wearable technology and patient-reported questionnaires. J Orthop 20:236-239

15. Mouton C, Hirschmann MT, Ollivier M, Seil R, Menetrey J (2020) COVID-19-ESSKA guidelines and recommendations for resuming elective surgery. J Exp Orthop 7:28
16. O'Connor CM, Anoushiravani AA, DiCaprio MR, Healy WL, Iorio R (2020) Economic recovery after the COVID-19 pandemic: resuming elective orthopedic surgery and total joint arthroplasty. J Arthroplasty. https://doi.org/10.1016/j.arth.2020.04.038

17. Oikonomou N, Tountas Y (2011) The Greek economic crisis: a primary health-care perspective. Lancet 377:28-29

18. Oussedik S, Zagra L, Shin GY, D'Apolito R, Haddad FS (2020) Reinstating elective orthopaedic surgery in the age of COVID- 19. Bone Joint J. https://doi.org/10.1302/0301-620x.102b7.bjj-202008081-4

19. Stucki G, Meier D, Stucki S, Michel BA, Tyndall AG, Dick W et al (1996) Evaluation of a German version of WOMAC (Western Ontario and McMaster Universities) Arthrosis Index. Z Rheumatol 55:40-49

20. Tegner Y, Lysholm J (1985) Rating systems in the evaluation of knee ligament injuries. Clin Orthop Relat Res 198:43-49

21. Thaler M, Khosravi I, Hirschmann MT, Kort NP, Zagra L, Epinette JA et al (2020) Disruption of joint arthroplasty services in Europe during the COVID-19 pandemic: an online survey within the European Hip Society (EHS) and the European Knee Associates (EKA). Knee Surg Sports Traumatol Arthrosc. https ://doi.org/10.1007/s00167-020-06033-1

22. Ware J Jr, Kosinski M, Keller SD (1996) A 12-Item Short-Form Health Survey: construction of scales and preliminary tests of reliability and validity. Med Care 34:220-233

23. Ware JE Jr, Sherbourne CD (1992) The MOS 36-item short-form health survey (SF-36). I. Conceptual framework and item selection. Med Care 30:473-483

24. Wirth B, Meier N, Koch PP, Swanenburg J (2013) Development and evaluation of a German version of the Tegner activity scale for measuring outcome after anterior cruciate ligament injury. Sportverletz Sportschaden 27:21-27

25. Yang BM, Prescott N, Bae EY (2001) The impact of economic crisis on health-care consumption in Korea. Health Policy Plan $16: 372-385$

Publisher's Note Springer Nature remains neutral with regard to jurisdictional claims in published maps and institutional affiliations. 